\author{
E. ROCA ${ }^{1,2}$, C. LOMBARDI ${ }^{3}$
}

\title{
Covid-19: less bronchial airways, more lung alveolar space and blood ways
}

${ }^{1}$ Covid-19 Unit, Division of Neurosurgery, Department of Head and Neck, Poliambulanza Foundation Hospital, Brescia, Italy

${ }^{2}$ Technology for Health, PhD program, University of Brescia, Brescia, Italy

${ }^{3}$ Departmental Unit of Pulmonary and Allergic Respiratory Diseases, Poliambulanza Foundation Hospital, Brescia, Italy

\section{KEY WORDS}

Asthma; Covid-19; SARS-CoV-2;

thromboembolism; endothelial dysfunction.

\author{
Corresponding author \\ Elena Roca \\ Covid-19 Unit \\ Division of Neurosurgery \\ Department of Head and Neck \\ Poliambulanza Fundation Hospital \\ via Leonida Bissolati 57 \\ 25124 Brescia, Italy \\ ORCID ID: 0000-0002-2314-7000 \\ E-mail: rocaelena@gmail.com
}

Doi

10.23822/EurAnnACI.1764-1489.205

To the Editor,

we report our experience in Brescia during the pandemic of Covid-19 by focusing on asthmatic patients affected by this infection, because we believe it is an interesting aspect to investigate. In late December 2019 many Chinese patients were hospitalized with an initial diagnosis of pneumonia of unknown aetiology. Subsequently, in the city of Wuhan, a new coronavirus was identified as responsible: severe acute respiratory syndrome coronavirus 2 (SARS$\mathrm{CoV}-2$ ) and the related disease (COVID-19), named by WHO on February 11, 2020, has then developed into a global pandemic. Patients affected by COVID-19 suffer with fever, upper respiratory airways symptoms and pneumonia with the risk of respiratory failure and death; others symptoms are described too like ageusia, anosmia and gastrointestinal symptoms.

In a recent article, by Richardson et al, the characteristics, comorbidities and outcomes of 5700 patients hospitalized with COVID-19 are analysed (1).

Analysing the data reported by the authors on comorbidities it is interesting to note that there is no increase in hospitalizations of asthmatic patients: the reported percentage $(9 \%)$ in the table is in line with the annual American one (2).

Considering the COVID-19 characteristics, it should be expected to observe an increase of asthma exacerbations and hospitalizations, a typical and frequent consequences of viral respiratory infections, in patients with asthma comorbidity as happened in the pandemic of Swine Influenza A (H1N1) and in the Middle East Respiratory Syndrome (MERS).

Instead, as in the United States even in our hospital, the percentage of asthmatic patients was in line with the Brescia (Lombardia Region, Northern of Italy) prevalence typical of this season (3). In the months of the pandemic explosion, especially from January to March 2020, our hospital in Brescia did not show an increase in emergency room admission of asthmatic patients if we compare these data with those relating to the same period of the previous year (January to March 2019). There has been no increase of these cases: 199 patients in the year 2020 vs 218 in the year 2019 (table I). 
Furthermore, according to these data, the prevalence of asthmatics in COVID-19 positive patients admitted to our hospital was lower than in the general population (20 patients out of a total of 1043 positive hospitalised patients $(1.92 \%), \mathrm{F} / \mathrm{M}=$ 12/8; age range: $41-77$ years; mean age: 61 years). The asthma prevalence in Italy is $5-6 \%$ in the general population.

All this astonish considering that normally there is a close association between asthma and viral respiratory infections, usually with an increased frequency and severity of lower respiratory tract infection in asthmatic patients and that asthma predisposes to airway dysfunction due to chronic inflammation (4).

These data are even more significant if we consider that the pandemic coincided with the seasonal increase in pollen counts in the Northern of Italy; despite this there were no asthmatic exacerbations in COVID-19 positive patients.

Therefore, it appears that asthma is not a risk factor in the development of COVID-19 and that SARS-Cov-2 does not aggravate asthma.

A possible explanation is that therapies used by asthmatic patients can reduce the risk of infection or developing symptoms: inhaled steroids, alone or in combination with bronchodilators, could promote the suppression of viral replication and of the production of cytokines responsible for the inflammatory storm. 15 of our 20 patients were being treated with Long-acting beta-agonists (LABAs) in association with Inhaled corticosteroids (ICSs).

Another interesting aspect is that asthma could protect against COVID-19, perhaps through a different pattern of immune response elicited by the chronic disease itself. In particular a different immune response, in patients with allergic asthma, could lead to a higher production of natural antibodies than non-allergic one (5). Is therefore COVID-19 only an exclusively airway disease? It is probably a more complex entity selectively affecting the alveolar space and the blood vessels (6).

To confirm this hypothesis there are several studies in the literature demonstrating the important role of thromboembolism (7). SARS-

Table I - Patients admitted to emergency room at our hospital in the months January-March 2019 compared to 2020, stratified by asthma and thromboembolic disease.

\begin{tabular}{lcccc}
\hline & \multicolumn{2}{c}{ Asthma } & \multicolumn{2}{c}{ Thromboembolism } \\
\hline January & $\mathbf{2 0 1 9}$ & $\mathbf{2 0 2 0}$ & $\mathbf{2 0 1 9}$ & $\mathbf{2 0 2 0}$ \\
\hline February & 79 & 72 & 3 & 3 \\
\hline March & 87 & 65 & 7 & 5 \\
\hline Total* & 52 & 62 & 4 & 24 \\
\hline
\end{tabular}

${ }^{*}$ Asthma p-value $=0.104741 ;{ }^{*}$ Thromboembolism p-value $=$ the standardized value of the sample is 9.36 , thus the associated $\mathrm{p}$-value is smaller than $10^{-16}$.
CoV-2 appears to have direct and indirect effects to thrombotic events, both in the venous and arterial circulations, so much so that haemostatic alterations, including disseminated intravascular coagulation (DIC), have been reported in patients with COVID-19. The authors claim that endothelial dysfunction, platelet activation, stasis and excessive inflammation may be the major factors implicated in the pathogenesis of thromboembolism (7).

Furthermore, by checking the accesses for thromboembolism at our hospital in the first three months of the year 2020 and comparing them with the same ones in 2019, we noticed an increase in cases: 32 patients (of which 25 patients positive for SARS-CoV2 on nasopharyngeal swab) compared to 14 . Statistical analysis of data confirmed that this increase was very significant: the standardized value of the sample is 9.36 , thus the associated p-value is smaller than $10^{-16}$ (table I).

The thromboembolic phenomenon is responsible for the high mortality rate of COVID-19 showing that, probably, this is the major complication due to SARS-CoV-2 infection.

Therefore, it is possible to hypothesize that COVID-19 probably represents a more complex pathology than an airway infection only: it involves less airways and much more alveolar spaces and blood vessels.

\section{Conflict of interests}

The authors declare that they have no conflict of interests.

\section{References}

1. Richardson S, Hirsch JS, Narasimhan M, et al. Presenting Characteristics, Comorbidities, and Outcomes Among 5700 Patients Hospitalized With COVID-19 in the New York City Area. JAMA 2020;323(20):2052-9. Erratum in: JAMA 2020;323(20):2098.

2. CDC.gov. CDC-Asthma-Data and Surveillance-AsthmaSurveillance Data, 2018. Available at: http://www.cdc.gov/asthma/asthmadata.htm. Last access date: 06/13/2019.

3. Cazzola M, Puxeddu E, Bettoncelli G, et al. The prevalence of asthma and COPD in Italy: a practice-based study. Respir Med 2011;105(3):386-91.

4. Papi A, Brightling C, Pedersen SE, Reddel HK. Asthma. Lancet 2018;391(10122):783-800.

5. Halpin DMG, Faner R, Sibila O, Badia JR, Agusti A. Do chronic respiratory diseases or their treatment affect the risk of SARSCoV-2 infection? Lancet Respir Med 2020;8(5):436-8.

6. Yao XH, Li TY, He ZC, et al. [A pathological report of three COVID-19 cases by minimal invasive autopsies]. Zhonghua Bing Li Xue Za Zhi 2020;49(5):411-7.

7. Bikdeli B, Madhavan MV, Jimenez D, et al; Global COVID-19 Thrombosis Collaborative Group, Endorsed by the ISTH, NATF, ESVM, and the IUA, Supported by the ESC Working Group on Pulmonary Circulation and Right Ventricular Function. COVID-19 and Thrombotic or Thromboembolic Disease: Implications for Prevention, Antithrombotic Therapy, and Follow-Up: JACC State-of-the-Art Review. J Am Coll Cardiol 2020;75(23):2950-73. 